\title{
NICA fixed target mode: soft jet studies in the relative 4-velocity space
}

\author{
V.A. Okorokov a \\ National Research Nuclear University MEPhI (Moscow Engineering Physics Institute), \\ Kashirskoe Shosse 31, 115409 Moscow, Russian Federation
}

Received: 27 September 2015 / Revised version: 7 February 2016

\begin{abstract}
Experimental results obtained by studying the properties of soft jets in the 4-velocity space at $\sqrt{s} \sim 2-20 \mathrm{GeV}$ are presented. The changes in the mean distance from the jet axis to the jet particles, the mean kinetic energy of these particles, and the cluster dimension in response to the growth of the collision energy are consistent with the assumption that quark degrees of freedom manifest themselves in processes of pion-jet production at intermediate energies. The energy at which quark degrees of freedom begin to manifest themselves experimentally in the production of soft pion jets is estimated for the first time. The estimated value of this energy is $2.8 \pm 0.6 \mathrm{GeV}$. The suggestions are made for future investigations on NICA.
\end{abstract}

PACS. 13.85.Hd Inelastic scattering: many-particle final states

\section{Introduction}

At the present time, the decision of the problem of confinement and the study of transition from meson-baryon degrees of freedom to quark-gluon ones is one of the most important (and, at the same time, most difficult) tasks of the world research programm in the field of strong interactions. The nature of confinement of color degrees of freedom (quark and gluons) and, correspondingly, the possible phase transitions in strongly interacting matter is not completely clear so far. Observation of hadron jets at high energies is one of the most important and evident experimental manifestation of quark-gluon degrees of freedom. At present, an open question is where is the low boundary on energy starting with which the color degrees of freedom should be taken into account for the description of processes of multiparticle production. Obviously, the jet structure of events displays itself more clearly at high initial energies $(\sqrt{s})$ than at intermediate ones. But in spite of this feature it seems that the application of collective characteristics of multiparticle final state can be useful in the collision energy domain $\sqrt{s} \simeq 2-20 \mathrm{GeV}$ both for the study of transition from the predominance of mesonbaryon degrees of freedom to the quark-gluon ones and for qualitative estimation of low boundary (for initial energy) experimental manifestation of quark degrees of freedom in soft jet production. In various fields of physics the onset of manifestation of new degrees of freedom and transition processes is accompanied by the presence of selfaffine and fractal properties in collective effects. There-

\footnotetext{
a e-mail: VAOkorokov@mephi.ru; Okorokov@bnl.gov
}

fore, the precise measurements of collective and geometric (fractal-like) properties of soft pion jets in the NICA energy domain can give a new important information about hadronization mechanisms, behavior of quantum systems in the nonperturbative region and transition to manifestation of quark degrees of freedom in collective phenomena.

\section{Method and variables}

Traditional collective characteristics used for the study of the event shape 1 are not relativistically invariant. This introduces some additional kinematic uncertainties, for example, in the choice of the center-of-mass system for reactions with atomic nuclei. A relativistic-invariant method was proposed in 2 for studying collective effects in the case where particle-beam interaction with a target leads to the formation of a multiparticle final state in the reaction $\mathrm{b}+\mathrm{t} \rightarrow 1+2+\ldots$ Special features of this method in the case of the production of two jets were considered in detail in $[3,4,5,6,7$. In that case, secondary particles refer to the region of target (beam) fragmentation if $X_{\mathrm{t}}^{k} \geq(\leq) \tilde{X} \cap X_{\mathrm{b}}^{k} \leq(\geq) \tilde{X}$, where $X_{\mathrm{p}}^{k}=$ $\left[m_{k}\left(U_{k} U_{\mathrm{r}}\right)\right]\left[m_{\mathrm{p}}\left(U_{\mathrm{p}} U_{\mathrm{r}}\right)\right]^{-1}, \mathrm{p}, \mathrm{r}=\mathrm{t}, \mathrm{b} ;$ and $\mathrm{p} \neq \mathrm{r} ; m_{k}$ is the mass of the $k^{\text {th }}$ secondary particle; $m_{\mathrm{t} / \mathrm{b}}$ is the mass of the target/beam particles; $U_{k}=P_{k} / m_{k}$ is the 4-velocity; $k=\mathrm{t}, \mathrm{b}, 1,2, \ldots$; and $\tilde{X}=0.1-0.2$ is some boundary value which is determined empirically. The basic quantities which the probability distributions (cross sections) depend upon are non-dimensional positive relativistic invariant quantities $b_{i k}=-\left(U_{i}-U_{k}\right)^{2}$, where $i, k=\mathrm{t}, \mathrm{b}, 1,2, \ldots$ 
[2]. As seen the observables $b_{i k}$ mean the squares of relative distances in the four-velocities space. The comparison of this method for distinguishing of some particle groups in the space of four-dimensional velocities with other present non-invariant (traditional) methods allows to name these separate groups as jets [8]. One of the most important observables of this approach is defined as $b_{k}=-\left(V-U_{k}\right)^{2}, k=\mathrm{t}, \mathrm{b}, 1,2, \ldots$, where $V=U_{J} /\left|U_{J}\right|$, $U_{J}=\sum_{i=1}^{N} U_{i}$ and $N$ is the number of particles in the considered fragmentation region which satisfies all cuts and is involved in the analysis [2,9. The quantity $b_{k}$ is the square of the distance of the $k^{\text {th }}$ particle from the jet axis $V$ in the space of $U_{k}$. The "temperature" defined as the mean kinetic energy of particles in the jet rest frame, $\left\langle T_{k}\right\rangle$, is estimated on the basis of the invariant functions $F\left(b_{k}\right)$ that, for pion jets, have the form [4] $F\left(b_{k}\right)=(\varepsilon / N) d N / d b_{k}$, where $\varepsilon \equiv 4 /\left[m_{\pi}^{2} b_{k} \sqrt{1+4 / b_{k}}\right]$, and which characterize the invariant cross section [9]. In [10, it was proposed to study the geometric properties of jets in the $U_{k}$ space with the aid of the cluster dimension $D$ defined on the basis of the relation between the number of particles in the jet being considered, $N\left(b_{k}\right)$, and its radius: $N\left(b_{k}\right) \propto b_{k}^{D / 2}$. Noninteger value of the cluster dimension can be considered as characteristic signature of manifestation of fractal-like properties 11. For most complex distributions the multifractal structure can appear and the cluster dimension be a function of jet radius for such case: $D=D\left(b_{k}\right)$. Thus $D$ is the qualitative parameter reflecting the features of particle distribution in phase space. The set of observables $\mathcal{G} \equiv\left\{\mathcal{G}_{i}\right\}_{i=1}^{3}=\left\{\left\langle b_{k}\right\rangle,\left\langle T_{k}\right\rangle, D\right\}$ characterizing the geometry and dynamics of the final-state production in the 4velocity space is under consideration and is proposed for future investigation on NICA.

\section{Recent results}

Figure 1 gives the parameters from the set $\mathcal{G}$ versus $\sqrt{s}$ for various interactions at $\tilde{X}=0.1$ (a, c, e) and $\tilde{X}=0.2$ $(\mathrm{b}, \mathrm{d}, \mathrm{f})$. The experimental-data array from [6, 7] is under study and various samples of these data are approximated by the functions

$$
\begin{aligned}
\mathcal{G}_{i} & =a_{1}\left(\sqrt{s / s_{0}}-a_{2}\right)^{a_{3}}, \quad \sqrt{s / s_{0}} \geq a_{2} ; \\
\mathcal{G}_{i} & =a_{1}+a_{2} \ln \left(s / s_{0}\right) .
\end{aligned}
$$

where $i=1-3$ and $s_{0}=1 \mathrm{GeV}^{2}$. One can see a qualitatively similar character of the energy dependence for all parameters from the set $\mathcal{G}$ at the respective values of $\tilde{X}$. Figure 1 shows that, for any value of $i$, the dependencies $\mathcal{G}_{i}(\sqrt{s})$ exhibit a change in behavior in the region around $\sqrt{s} \simeq 3 \mathrm{GeV}$ for all interaction types, with the exception of $h A$, at any value of $\tilde{X}$, and this confirms the hypothesis put forth in [4] that dynamical interaction regimes undergo a change for $\sqrt{s}<3-4 \mathrm{GeV}$.

In the region of $\sqrt{s}<4 \mathrm{GeV}$, experimental dependencies $\mathcal{G}_{i}(\sqrt{s}), i=1-3$, for all interaction types, with the exception of $h A$, are approximated by the function in (1).
The results of samples that combine the fragmentation regions are shown by dotted lines. One can see that the function in (1) agrees qualitatively with experimental data at all of the $\tilde{X}$ values considered here. The parameter $a_{2}$ in (1) can be put in correspondence with the energy $\sqrt{s_{c}}$ at which quark-gluon degrees of freedom begin to manifest themselves in the production of soft pion jets. The values of $\sqrt{s_{c}}$ for the members of $\mathcal{G}$ are given in the Table 1. The estimates of $\sqrt{s_{c}}$ for $\left\langle T_{k}\right\rangle$ and $D$ agree well with each other but exceed the values of $\sqrt{s_{c}}$ obtained earlier in [5] on the basis of the $\left\langle b_{k}\right\rangle(\sqrt{s})$ dependence. This difference may be due both to physical reasons that lead to a sharper growth of $\left\langle T_{k}\right\rangle$ and $D$ in relation to $\left\langle b_{k}\right\rangle$ and to a smaller size of the experimental-data samples in the case of the first two parameters. In the energy region being considered, an approximation by the function in (1) is also constructed for individual fragmentation regions. There is a substantial improvement of the quality of fits for any fragmentation region and any value of $\tilde{X}\left(\chi^{2} / \mathrm{ndf} \sim 3-5\right)$. Statistically acceptable values of $\chi^{2} /$ ndf could be obtained for $45 \%$ of the samples 6,7]. Numerical values found for $\sqrt{s_{c}}$ from a fit to $\mathcal{G}_{i}(\sqrt{s})$ for $\sqrt{s}<4 \mathrm{GeV}$ are summarized in the Table 1, the values corresponded to the fits of $\left\langle b_{k}\right\rangle$ energy dependence being taken from [5]. The values of $\sqrt{s_{c}}$ for the remaining two parameters from $\mathcal{G}$ are presented in the first lines for $\left\langle T_{k}\right\rangle$ and $D$. Taking into account the sizes of samples of the available experimental data and the behavior of $\left\langle T_{k}\right\rangle(\sqrt{s})$ and $D(\sqrt{s})$, we can approximate the dependencies in question by the function in (11) for $\sqrt{s}>2$ $\mathrm{GeV}$. A fit was constructed both for global samples and for the regions of target and beam fragmentation. For the first case, the fitted results are shown by the dashed curves in Figs. 1 - f. For $\left\langle T_{k}\right\rangle$, agreement between the approximation by the function in (11) and experimental data is only qualitative in the case of $\tilde{X}=0.1$ (see Fig. 1 1 ), since the fit quality is substantially poorer in that case than in the case of $\tilde{X}=0.2$ (see Fig. 11d). For $D$, the fit quality is indicative of only qualitative agreement between the function in (11) and experimental data at either value of $\tilde{X}$ (see Figs. 1, f) 6,7]. In the Table 1, the values obtained for $\sqrt{s_{c}}$ from a fit in the region of $\sqrt{s}>2 \mathrm{GeV}$ for global samples and for individual samples in the target- and beam fragmentation regions are given in the second rows for $\left\langle T_{k}\right\rangle$ and $D$. The experimental results for the traditional definition of the thermal freeze-out temperature $(T)$ obtained for $p p$ collisions at $\sqrt{s} \approx 6-18 \mathrm{GeV}$ [12] agrees quite reasonably with $\left\langle T_{k}\right\rangle(\sqrt{s})$ at soft $\tilde{X}=0.1$ (see Fig. 1k) in both the functional behavior and the magnitude. As consequence, the thermal freeze-out temperatures 12 are some smaller than the mean "temperatures" of pions in jets at hard cut $\tilde{X}=0.2$ and close energies. It should be noted $T(\sqrt{s})$ calculated within the framework of the self-similarity approach assumes the sharp decrease at $\sqrt{s}<5 \mathrm{GeV}$ and is almost flat at larger energies 13. Such behavior of $T(\sqrt{s})$ agrees well with the features of $\left\langle T_{k}\right\rangle(\sqrt{s})$ (Fig. 1 $k$, d) and confirms the hypothesis with regard to the changing of the dynamical regimes of multiparticle production at $\sqrt{s} \sim 5$ $\mathrm{GeV}$ [4]. 


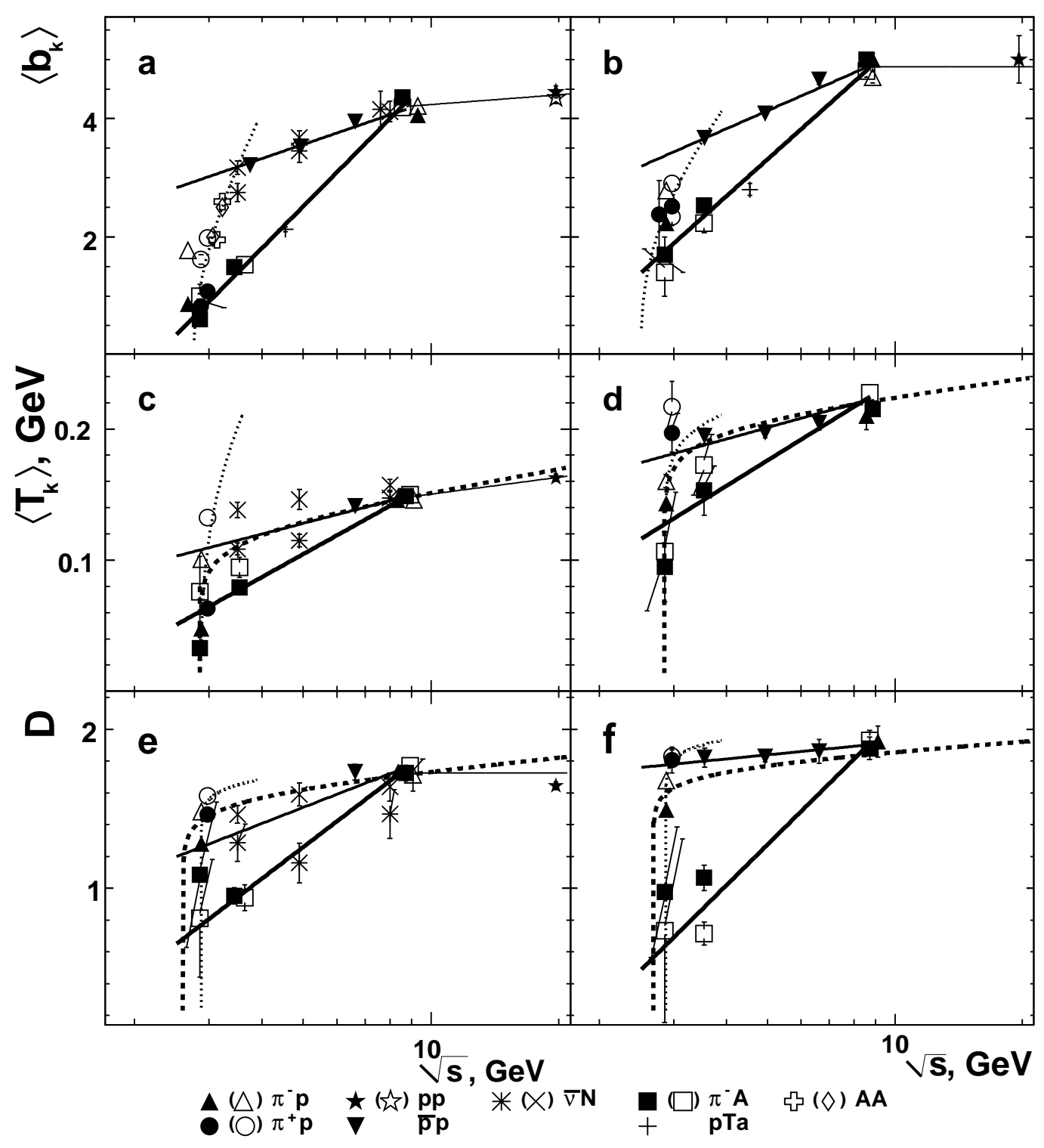

Fig. 1. Dependence of parameters from set $\mathcal{G}$ on $\sqrt{s}$ at $\tilde{X}=0.1$ (a, c, e) and 0.2 (b, d, f) in the region of target (beam) fragmentation [7. The dotted line corresponds to the approximation of data in the region of $\sqrt{s}<4 \mathrm{GeV}$ by the function (1), while the dashed lines in Figs. c - f represent the approximation of all available experimental data by the function (1). The solid lines stand for the results obtained by fitting the logarithmic function (2) to a global sample of $h h$ and $\bar{\nu} N$ reactions for $4 \leq \sqrt{s}<9 \mathrm{GeV}$ (line with moderate thickness), data on $h A$ reactions (thick line), and data on $h h$ and $h A$ interactions for $\sqrt{s}>8 \mathrm{GeV}$ (thin line).

One can see that the values of $\sqrt{s_{c}}$ are reasonably consistent for the different $\tilde{X}$ values and fragmentation regions. Thus, this investigation extended for the whole set $\mathcal{G}$ renders the results obtained earlier in 4,5 more reliable and furnishes an additional argument in support of the hypothesis that a changeover of dynamical interaction regimes occurs because of the onset of the exper- imental manifestation of quark degrees of freedom in the production of soft pion jets at $\sqrt{s} \sim 3 \mathrm{GeV}$. This entails the respective transition from the description of the processes in question in terms of baryon-meson degrees of freedom to the use of quark-gluon degrees of freedom. The lower boundary of the energy corresponding to the onset of the experimental manifestation of quark degrees 
V.A. Okorokov: NICA fixed target mode: soft jet studies in the relative 4-velocity space

Table 1. Values of $\sqrt{s_{c}}$ (in GeV units) at the boundary values of $\tilde{X}=0.1$ and 0.2 [

\begin{tabular}{ccccccc}
\hline Parameter & \multicolumn{2}{c}{ Global sample } & \multicolumn{2}{c}{ Target fragmentation } & \multicolumn{2}{c}{ Beam fragmentation } \\
\cline { 2 - 7 } from set $\mathcal{G}$ & 0.1 & 0.2 & 0.1 & 0.2 & 0.1 & 0.2 \\
\hline$\left\langle b_{k}\right\rangle$ & $2.76 \pm 0.01$ & $2.51 \pm 0.03$ & $2.82 \pm 0.02$ & $2.46 \pm 0.04$ & $2.43 \pm 0.04$ & $2.5 \pm 0.3$ \\
$\left\langle T_{k}\right\rangle$ & $2.877 \pm 0.001$ & $2.865 \pm 0.007$ & $2.75 \pm 0.04$ & $2.865 \pm 0.007$ & $2.877 \pm 0.001$ & - \\
& $2.854 \pm 0.001$ & $2.854 \pm 0.001$ & $2.853 \pm 0.001$ & $2.854 \pm 0.001$ & $2.877 \pm 0.001$ & $2.854 \pm 0.001$ \\
$D$ & $2.875 \pm 0.001$ & $2.875 \pm 0.001$ & $2.875 \pm 0.004$ & $2.877 \pm 0.001$ & $2.78 \pm 0.04$ & - \\
& $2.60 \pm 0.03$ & $2.68 \pm 0.03$ & $2.854 \pm 0.002$ & $2.858 \pm 0.001$ & $2.9 \pm 0.4$ & $2.874 \pm 0.001$ \\
\hline
\end{tabular}

of freedom in the production of soft pion jets was estimated quantitatively for the first time. The interval in which the estimates of this parameter are contained and which is matched with the results for all of the collective features under consideration from $\mathcal{G}$ (see Table 1) is $\sqrt{s_{c}}=2.43-2.90 \mathrm{GeV}$. Treating the set of estimates of $\sqrt{s_{c}}$ from the Table 1 as a sample of independent measurements, one can obtain $\left\langle\sqrt{s_{c}}\right\rangle=2.78 \pm 0.14 \mathrm{GeV}$. On the other hand, allowance for the amplitude of changes in $\sqrt{s_{c}}$ within one standard deviation (see Table 1) yields the interval $\left[\left(\sqrt{s_{c}}\right)_{\min } ;\left(\sqrt{s_{c}}\right)_{\max }\right]$ for which the choice of midpoint and mean deviation makes it possible to obtain an estimate of $\left\langle\sqrt{s_{c}}\right\rangle=(2.8 \pm 0.6) \mathrm{GeV}$ [6,7]. A universal lower boundary for the manifestation of jet geometry for final states was qualitatively estimated in [14] at $\sqrt{s_{l}} \sim 3 \mathrm{GeV}$ for multiparticle-production processes. This value agrees with $\sqrt{s_{c}}$ with allowance for the errors. Possibly, the parameters $\sqrt{s_{l}}$ and $\sqrt{s_{c}}$ characterize the same physical effect - the onset of experimental manifestations of quark degrees of freedom in soft processes of the multiparticle production of secondaries and, hence, a manifestation of the jet structure of the event being considered. Thus, the results obtained by using traditional and four-dimensional collective variables agree reasonably and supplement each other.

Taking into account the results for $\left\langle b_{k}\right\rangle(\sqrt{s})$ in the region of $\sqrt{s}>3.5 \mathrm{GeV}$ [4, 5] and the behavior of $\left\langle T_{k}\right\rangle(\sqrt{s})$ and $D(\sqrt{s})$ in the above range of $\sqrt{s}$, we approximated by the function (2) the samples taken for $\left\langle T_{k}\right\rangle$ and $D$ and summed over the fragmentation regions. The numerical values of the fit parameters and the respective detailed discussion are presented in 6. From Fig. 1] one can see that, for $3.5<\sqrt{s}<9 \mathrm{GeV}$, the values of $\mathcal{G}_{i}, i=1-3$, grow faster for $h A$ reactions than for a nucleon target. Analyzing the results obtained for $\left\langle T_{k}\right\rangle$ and $D$ in the range of $\sqrt{s} \sim 3-5 \mathrm{GeV}$ and taking into account large statistical errors in $h A$ data, one can extend the conclusion concerning the effect of nuclear matter on the properties of soft pion jets 3 to the whole set $\mathcal{G}$. Approximations for $\left\langle T_{k}\right\rangle(\sqrt{s})$ and $D(\sqrt{s})$ in the region of $\sqrt{s}>8 \mathrm{GeV}$ are possible only in the case of a soft limit on $\tilde{X}$ (see Figs. $1 \mathrm{1}, \mathrm{e})$. In view of the scantiness of the available data, we cannot rule out definitively a weak logarithmic growth for $D$ in accordance with Eq. (2). Thus, we see that, for all $i$, the dependencies $\mathcal{G}_{i}(\sqrt{s})$ admit a universal approximation in the form (2) for a broad class of interactions at c.m. energies in the region of $\sqrt{s}>3.5 \mathrm{GeV}$ and for both $\tilde{X}$ values considered in the present study.

\section{Further advancement}

It is noteworthy, that agreement of the results of investigation of all parameters from the set $\mathcal{G}$ with one another and the self-consistency of a global analysis of the properties of soft pion jets in $U_{k}$ space renders the hypothesis of the onset of experimental manifestation of quark degrees of freedom in processes of the production of soft pion jets at $\sqrt{s} \sim 3 \mathrm{GeV}$ more plausible. However in order to test the above hypothesis it would be reasonable to perform additional investigations in the range of $\sqrt{s} \sim 2-20$ $\mathrm{GeV}$. Furthermore the limited samples of experimental results for $\left\langle T_{k}\right\rangle$ and $D$ and significant errors especially for $\sqrt{s} \sim 3 \mathrm{GeV}$ allow a semi-qualitative analysis only for these observables. Therefore additional high-statistic investigations on the NICA with various beams would be important for better understanding of the dynamic and geometric features of the production of soft pion jets.

The separation of various dynamics of jet production is a difficult task especially in the non-perturbative region of $\sqrt{s}$. The study of traditional collective observables at such $\sqrt{s}$ allows the conclusion for event shape mostly 1 . Within the approach in question, the range $10^{-2} \leq b_{i k} \sim 1$ corresponds to the transition from the domain of dominance of meson-baryon degrees of freedom to the region where the internal structure of colliding particles and, as a consequence, quark-gluon degrees of freedom become essential in the processes of secondary particle jet production 1 . The estimations for boundaries of various dynamic domains in terms of $b_{i k}$ seem valid for $b_{k}$, i.e. when the jet axis corresponds to the "reference" $i$ th particle. That is why the distributions on $b_{k}$ are usually studied. The approach under discussion allows the separation, at least, at qualitative level different dynamics of soft jet production. This advantage of the relativistically invariant method is important for intermediate $\sqrt{s}$ namely. Therefore approaches for the analysis of jet production based on traditional collective characteristics and on the set $\mathcal{G}$ are complementary to each other at intermediate $\sqrt{s}$. Complete investigation of jet production seems important especially at NICA for the energy region which corresponds to the

\footnotetext{
1 One needs to note that the boundary values for $b_{i k}$ indicated above are qualitative phenomenological estimations.
} 
transition from the dominance of meson-baryon degrees of freedom to quark-gluon ones.

Another suggestion is the study of the behavior of $\alpha_{S}$ - the effective strong coupling constant - in the deeply non-perturbative region of $\sqrt{s}$. In the lowest order of the renormalization group equation (RGE) the following relation was derived $\alpha_{S} \propto 1 / \ln b_{i k}$, where $i=\mathrm{t} / \mathrm{b}, 15$. The possible exact relation between $b_{k}$ and $\alpha_{S}$ requires an additional rigorous substantiation and careful derivation. Here one can note only that jets consist of particles with close masses (pions) in the present study and there is the relation $\left\langle b_{k}\right\rangle=2\left(\left\langle M_{J}\right\rangle /\left\langle n_{J}\right\rangle m_{h}-1\right)$ between the geometry quantity of jet in $U_{k}$ space and mean invariant mass $\left(M_{J}\right)$ of jet of hadrons with equal masses $m_{h} 16$, 17. Here $\left\langle n_{J}\right\rangle$ is the mean multiplicity of particles inside the jet, the averaging is taken over particles in the event and over events in the sample in the l.h.s.; and over event ensemble in the r.h.s. The appropriate choice of the energy scale $Q$ in the RGE is non-trivial for hadronic processes especially in the deeply non-perturbative region. By analogy with collider experiments $18,19,\left\langle M_{J}\right\rangle$ is chosen as $Q$ for some reaction at fixed $\sqrt{s}$ within the framework of the method under study. Then based on the 20 one can derive the relation

$$
\alpha_{S}=\left(b_{0} \zeta\right)^{-1}, \quad \zeta \equiv 2 \ln \left[\left(0.5\left\langle b_{k}\right\rangle+1\right)\left\langle n_{J}\right\rangle m_{h} \Lambda^{-1}\right]
$$

where $b_{0}=\left(33-2 n_{f}\right) /(12 \pi)$ is the one-loop $\beta$-function coefficient, $n_{f}$ is the number of quark flavors considered as light, $\Lambda$ is the QCD parameter. The (3) demonstrates possible relation between soft jet characteristic $\left\langle b_{k}\right\rangle$ and strong coupling and gives indications on the additional advantage for study in non-perturbative regime at NICA. The estimations $\left\langle M_{J}\right\rangle \sim 1-2 \mathrm{GeV}$ obtained by the method under consideration for $\pi p$ reactions at $\sqrt{s} \sim 3 \mathrm{GeV} 17$ agree with the results at $\sqrt{s} \sim 9 \mathrm{GeV}[21$. Therefore the suggestion seems reasonable with regard to the validity of (3) in the NICA energy domain. It should be noted that there is one measurement of $\alpha_{S}$ in the deeply nonperturbative domain at $Q \sim 2 \mathrm{GeV}$, namely from $\tau$ decay [20]. Thus the suggested equation (3) can provide the important estimation of $\alpha_{S}$ at $Q \sim\left\langle M_{J}\right\rangle$ on the order of few $\mathrm{GeV}$ and verifies the validity the theoretical curve $\alpha_{S}(Q)$ at low energy scales, i.e. it allows the investigation of one of the fundamental properties of QCD in a most difficult domain for theoretical description.

\section{Summary}

Summarizing the foregoing, we can draw the following conclusions.

The dependencies $\mathcal{G}_{i}(\sqrt{s}), i=1-3$, exhibit qualitatively similar types of behavior, which admits a description in terms of power-law function for $\sqrt{s}<4 \mathrm{GeV}$ and in terms of a logarithmic function for $\sqrt{s}>3.5 \mathrm{GeV}$. The behavior of $\mathcal{G}_{i}(\sqrt{s})$ for all $i$ at $\sqrt{s} \sim 3 \mathrm{GeV}$ is likely to be due to the onset of an experimental manifestation of quark degrees of freedom in the production of soft pion jets and the respective transition from the description of the processes in question in terms of baryon-meson degrees of freedom to the use of quark-gluon degrees of freedom. A lower limit on the energy at which quark degrees of freedom begin to manifest themselves in the production of soft pion jets was estimated quantitatively for the first time. The result is $\left\langle\sqrt{s_{c}}\right\rangle=(2.8 \pm 0.6) \mathrm{GeV}$. The effect of nuclear matter on the dynamical and geometric properties of soft pion jets in the 4-velocity space was found in the range of $\sqrt{s} \sim 3-5 \mathrm{GeV}$.

The investigations of collective and geometric (fractal) properties in soft hadron and nuclear reactions at intermediate energies with high statistics at NICA can provide important progress for better a understanding of the deeply non-perturbative domain of strong interactions.

\section{References}

1. V.A. Okorokov, Int. J. Mod. Phys. A 27, 1250037 (2012).

2. A.M. Baldin, Sov. Phys. Dokl. 20, 418 (1975); ibid 29, 1031 (1984).

3. I.L. Kiselevich et al., Phys. At. Nucl. 57, 2140 (1994).

4. V.I. Mikhailichenko et al., Phys. At. Nucl. 62, 1665 (1999).

5. V.A. Okorokov et al., Phys. At. Nucl. 73, 1963 (2010).

6. V.A. Okorokov, Int. J. Mod. Phys. A 28, 1350150 (2013).

7. V.A. Okorokov, Phys. At. Nucl. 78, 415 (2015).

8. A.M. Baldin et al., Sov. J. Nucl. Phys. 44, 785 (1987).

9. A.M. Baldin and L.A. Didenko, Asymptotic properties of hadronic matter in the space of four-dimensional relative velocities. Lectures for young scientists 43, JINR, Dubna, 1987; A.M. Baldin and L.A. Didenko, Fortschr. Phys. 38, 261 (1990).

10. V.A. Okorokov et al., in Scientific session MEPhI-2000, MEPhI, V. 7, 2000, p. 218.

11. A.T. Fomenko, Visual geometry and topology: mathematical images in the real world, Moscow, MSU, 1998.

12. A.A. Abgrall et al., Eur. Phys. J. C74, 2794 (2014).

13. D.A. Artemenkov et al., Int. J. Mod. Phys. A30, 1550127 (2015).

14. V.A. Okorokov, Int. J. Mod. Phys. A27, 1250037 (2012); V.A. Okorokov, A.K. Ponosov, Phys. Atom. Nucl. 76, 1230 (2013).

15. A.M. Baldin, Nucl. Phys. A 434, 695c (1985).

16. V.G. Grishin, Quarks and hadrons in high energy particle interactions, Moscow, Energoatomizdat, 1988.

17. V.A. Okorokov, in XVIII International Baldin Seminar on High Energy Physics Problems "Relativistic Nuclear Physics and Quantum Chromodynamics", Eds. by A.N. Sissakian, V.V. Burov, A.I. Malakhov, JINR, Dubna, V. I, 2008, p. 154.

18. V.M. Abazov et al., Phys. Lett. B 718, 56 (2012).

19. S. Chatrchyan et al., Eur. Phys. J. C 73, 2604 (2013).

20. K.A. Olive et al., Chin. Phys. C38, 090001 (2014).

21. N.N. Badalyan et al., JINR Rap. Comm. 1, 27 (1996). 